\title{
Estudio de la fertilización química y orgánica y su efecto en el cultivo de Maíz (Zea mays.), en una comuna
}

\author{
Study of Chemical and Organic fertilization and its effect on the cultivation of Corn (Zea mays.), in the commune
}

Estudo da fertilização química e orgânica e seu efeito sobre o cultivo de milho (Zea mays.) em uma comuna

\author{
Juan Miguel Garcia Cabrera \\ juanmiguel213@hotmail.com \\ https://orcid.org/0000-0002-6334-7744
}

\author{
Carlos Alberto Castro Piguave \\ carlos.castro@unesum.edu.ec \\ https://orcid.org/0000-0003-3180-2359
}

\author{
Gaspar Martín Moreno Mera \\ gaspar.moreno2015@gmail.com \\ https://orcid.org/0000-0001-7201-7459
}

Universidad Estatal del Sur de Manabí, Manabí Ecuador

Artículo recibido 17 de febrero 2021 / Arbitrado y aceptado 24 de marzo 2021 / Publicado 04 de mayo 2021

\section{RESUMEN}

Basado al manejo de diversos tipos de fertilizantes de origen orgánico como químico empleados en el cultivo de Maíz (Zea Mays), se propuso determinar las diferencias dadas en el desarrollo morfológico del cultivo; e identificar la mejor nutrición que permita obtener mayores niveles de productividad $y$ que a su vez establecerá la relación costo-beneficio de los tratamientos establecidos. La metodología aplicada, permitió utilizar un modelo de bloques completamente al azar de cuatro repeticiones, los tratamientos basados en la fertilización fueron a partir de: Yara, Ecológico, Orgánico, Químico y un tratamiento testigo al que no se le realizo aplicación alguna de fertilizante; las variables consideradas dentro del proceso evaluativo fueron: altura de planta, diámetro de tallo, largo de hoja, inserción de mazorca, peso de mazorca, peso de tusa, peso de 100 granos, peso de granos, numero de hileras, disposición de las hileras y daños de la mazorca. Los resultados mostraron que el tratamiento Yara alcanzo una mayor productividad obteniendo así $38,31 \mathrm{~kg}$ en el peso de la parcela y que a su vez permitió evidenciar un mejor desarrollo morfológico en toda su etapa; estableciendo una altura de planta de $213,78 \mathrm{~cm}$; un diámetro de planta de $72 \mathrm{~mm}$ y una longitud de hoja de $101,58 \mathrm{~cm}$; lo cual permite contar con una mayor capacidad fotosintética gracias a su mayor cobertura foliar.

Palabras clave: Fertilización; Yara; Maíz; Desarrollo morfológico
ABSTRACT

Based on the management of various types of fertilizers of organic origin such as chemical used in the cultivation of Corn (Zea Mays), it was proposed to determine the differences given in the morphological development of the crop; and to identify the best nutrition that allows obtaining higher levels of productivity and which in turn will establish the cost-benefit relationship of the established treatments. The applied methodology allowed the use of a completely randomized block model of four repetitions, the treatments based on fertilization were based on: Yara, Ecological, Organic, Chemical and a control treatment to which no fertilizer was applied; the variables considered within the evaluation process were: plant height, stem diameter, leaf length, ear insertion, ear weight, cob weight, 100 grain weight, grain weight, number of rows, row arrangement and ear damage. The results showed that the Yara treatment reached a higher productivity thus obtaining $38.31 \mathrm{~kg}$ in the weight of the plot and that in turn allowed to show a better morphological development in all its stage; establishing a plant height of $213.78 \mathrm{~cm}$; a plant diameter of $72 \mathrm{~mm}$ and a blade length of $101.58 \mathrm{~cm}$; which allows for a greater photosynthetic capacity thanks to its greater foliar coverage.

Key words: Fertilization, Yara, Corn, Morphological development

\section{RESUMO}

Com base no manejo de diferentes tipos de fertilizantes de origem orgânica e química utilizados no cultivo do milho (Zea Mays), foi proposto determinar as diferenças dadas no desenvolvimento morfológico da cultura; e identificar a melhor nutrição que permita obter maiores níveis de produtividade e que, por sua vez, estabeleça a relação custo-benefício dos tratamentos estabelecidos. A metodologia aplicada permitiu o uso de um modelo de bloco completamente aleatório com quatro réplicas, os tratamentos baseados na fertilização foram baseados em: Yara, Ecológico, Orgânico, Orgânico, Químico e um tratamento de controle ao qual não foi feita aplicação de fertilizantes; as variáveis consideradas dentro do processo de avaliação foram: altura da planta, diâmetro do caule, comprimento da folha, inserção da espiga, peso da espiga, peso da espiga, peso do grão 100, peso do grão, número de filas, disposição das filas e danos na espiga. Os resultados mostraram que o tratamento Yara alcançou uma produtividade maior obtendo $38,31 \mathrm{~kg}$ no peso da parcela e que por sua vez permitiu demonstrar um melhor desenvolvimento morfológico ao longo de sua etapa; estabelecendo uma altura de planta de $213,78 \mathrm{~cm}$; um diâmetro de planta de $72 \mathrm{~mm}$ e um comprimento de folha de 101,58 cm; o que permite ter uma maior capacidade fotossintética graças a sua maior cobertura foliar.

Palavras-chave: Fertilização; Yara; Milho; Desenvolvimento morfológico 


\section{INTRODUCCIÓN}

La zona sur de Manabí, una zona totalmente maicera, y donde se encuentra el recinto Cantagallo, de la cual sus habitantes están acostumbrados a utilizar fertilizantes de mezcla física y fertilizantes con compuestos nitrógenos comunes en cultivos de maíz, lo cual hace que los híbridos de maíz usados en la zona no lleguen a expresar todo su potencial genético por una mala nutrición y una constante pérdida de nutrientes en el suelo. El objetivo principal de la investigación fue el verificar el punto más alto de productividad del cultivo de maíz usando un hibrido de alto rendimiento dando un mismo manejo agronómico con diferencia en el uso de sus fertilizantes, usando las diferentes alternativas de nutrición sean estas: abonos de mezcla química, abonos de mezcla física, abonos nitrogenados, y abonos orgánicos además de hacer un mezcla de fertilizantes químicos y orgánicos, evaluando los rendimientos al final del ciclo de producción, con el fin de sacar una comparación entre tratamientos, siendo este el punto clave de comprobación del aumento o disminución de rendimientos de acuerdo a la utilización de los diferentes nutriciones; además de su evaluación durante el desarrollo vegetativo de la planta.

Ya que los niveles de productividad de los cultivos de maíz en la zona de estudio, son bajos por muchos factores ya sean estos obviados o desconocidos por los agricultores; por lo que la investigación permitió a su vez aportar una solución al manejo idóneo de los cultivares de maíz en relación a la nutrición y de esta forma mejorando su productividad, ya que en la zona de estudio se están introduciendo diferentes híbridos de maíz pero no se explotan de la manera adecuada debido a que no existe un manejo técnico y muchas veces acorde en la nutrición del material empleado.

\section{MATERIALES Y MÉTODOS}

El estudio se llevó acabo en el periodo de Febrero a Julio del 2020, en la comuna Cantagallo, en la finca del señor Gordon Kane, misma que se encuentra ubicada a $36,1 \mathrm{~km}$ de la cabecera cantonal de Jipijapa, en la provincia de Manabí, (1); el lugar de estudio posee parámetros meteorológicas promedios de precipitación de 411 mm/año, al igual que temperaturas de $23,9^{\circ} \mathrm{C}$.

Se estableció un ensayo unifactorial, donde se emplearon los siguientes tratamientos de fertilización: YARA, ecológica, orgánica, química aplicando la tecnología del agricultor (Testigo 1) y una fertilización nitrogenada (Testigo 1).

Tabla 1. Delineamiento experimental de la investigación.

\section{DELINEAMIENTO EXPERIMENTAL}

$\begin{array}{ll}\text { Unidades o parcelas experimentales } & : 20 \\ \text { Número de repeticiones } & : 4 \\ \text { Número de tratamientos } & : 5 \\ \text { Hileras por parcela } & : 5 \\ \text { Hileras útiles } & : 3\end{array}$




\section{DELINEAMIENTO EXPERIMENTAL}

Hileras bordes por parcela $: 2$

Número de plantas por unidad experimental $: 125$

Número de plantas por parcela útil

$$
: 75
$$

Número de plantas evaluadas en parcela útil

$: 50$

Distancia entre hileras

: $0,80 \mathrm{~m}$

Distancia entre plantas

: 0,20 m

Distancia entre repeticiones

$: 2 \mathrm{~m}$

Distancia entre tratamientos

$: 1 \mathrm{~m}$

Longitud de parcela

$: 5 \mathrm{~m}$

Ancho de parcela

$: 4 \mathrm{~m}$

Área total de la parcela

: $20 \mathrm{~m} 2$

Área útil de la parcela

: $8 \mathrm{~m} 2$

Área útil del ensayo

: $160 \mathrm{~m} 2$

Área total del ensayo

: $624 \mathrm{~m} 2$

\section{Materiales}

Tabla 2. Materiales, herramientas e insumos utilizados en la investigación.

\begin{tabular}{lll}
\hline \multicolumn{1}{c}{ HERRAMIENTAS } & \multicolumn{1}{c}{ MAQUINARÍAS } & INSUMOS \\
\hline Machete & Sembradora & Cintas de riego por goteo $16 \mathrm{~mm}$ \\
Calibrador Vernier & Abonadora & Goteros convencionales de 3,0 l/h \\
Flexómetro & Bomba de mochila para fumigar & Semillas de maíz HIBRIDO ADV-9735 \\
Balanza gramera & & YARAVITA SINERGISE NP+Zn \\
Romana & & YARAVELA NITROMAG \\
& & YARA ZINTRAC \\
& YARA NITRODOBLE \\
& REMITAL M. 17-6-18-2 \\
& BIO REMEDY \\
& FUL HUMIX \\
& HUMUS \\
& FAST2AGRO \\
& GRANDSIL \\
& FOSSILL SHELL AGRO \\
& ABONO COMPLETO \\
& UREA \\
& Sacos de plástico de 25 kg \\
&
\end{tabular}




\section{Métodos}

\section{Manejo especifico de la investigación}

La investigación se realizó por toma de datos cada quince días durante el desarrollo vegetativo de la planta, durante todo ese proceso se hará la comparación de la altura de planta hasta llegar a la cosecha en donde se lo evaluó cuando la mazorca estaba en un estado maduro y totalmente seco para llegar a una relación de peso y así mismo se comparó sus pesos con el uso de los fertilizantes puestos a competir.

Limpieza de terreno: la limpieza de terreno se efectuó el 15 de enero de 2020, previamente con el desbroce de malezas a machete presentes en el terreno permitiendo contar con un suelo apto para el desarrollo experimental.

Arado, rastrado y surcado: se aplicó una labranza mínima utilizando maquinaria agrícola el 28 de enero de 2020, evitando alteraciones en la composición del suelo a utilizar en la investigación.

\section{Delimitación del terreno para la} experimentación: aquí se procedió a efectuar las respectivas mediciones y distribuciones de cada uno de los tratamientos a evaluar siendo esta labor el día 29 de enero del 2020.

Riego de pre siembra: se realizó un riego de pre siembra durante el 13 y 18 de febrero con el fin de mantener húmedo el terreno, conservando el mismo a capacidad de campo de acuerdo a lo establecido en el diseño agronómico de riego, basado a los datos meteorológicos recolectados de la zona; con ayuda de la estación meteorológica ubicada en los predios de la granja de la UNESUM ubicada en Cantagallo, permitiendo así una activación en la germinación de la semilla y evitando inconvenientes durante este proceso.

Siembra: se realizó la siembra el 20 de febrero a un distanciamiento de $80 \mathrm{~cm}$ entre surco y 20 $\mathrm{cm}$ entre plantas, obteniendo de esta manera 125 plantas por unidad experimental.

Control manual de malezas: se realizó un control de malezas de forma manual para evitar un cambio en los resultados por la influencia de herbicidas en el proceso de desarrollo fisiológico de la planta durante las fechas del 07, 14 y 21 de marzo.

Riego de los cultivos: se realizó un riego por goteo convencional, como se establece en la tabla 3; donde se identifican las necesidades hídricas del cultivo o diseño agronómico del sistema riego, optimizando el recurso agua y empleando la cantidad necesaria para el cultivo sin generar afectaciones en torno a este parámetro.

Tabla 3. Diseño agronómico de riego.

\begin{tabular}{cccccc}
\hline $\begin{array}{c}\text { Lámina de riego } \\
(\mathbf{m m})\end{array}$ & $\begin{array}{c}\text { Volumen de } \\
\text { agua }(\mathbf{m} 3)\end{array}$ & $\begin{array}{c}\text { Lámina de agua } \\
(\mathbf{m m})\end{array}$ & $\begin{array}{c}\text { Gasto de agua } \\
(\mathbf{l} / \mathbf{h r})\end{array}$ & $\begin{array}{c}\text { Tiempo de riego } \\
(\mathbf{h r})\end{array}$ & $\begin{array}{c}\text { Caudal requerido } \\
(\mathbf{m} 3 / \mathbf{h r})\end{array}$ \\
\hline 0,85 & 0,34 & 123 & 300 & 1 & 0,34 \\
\hline
\end{tabular}


Aplicación de los tratamientos: se evaluaron cinco tratamientos en donde se realizaron aplicaciones a los $0,8,15,21$, y 40 días como se detalla a continuación:

Tabla 4. Aplicación de tratamientos.

\begin{tabular}{|c|c|c|c|c|c|}
\hline $\begin{array}{c}\text { Días de } \\
\text { aplicación }\end{array}$ & $\begin{array}{c}\text { Fertilización } \\
\text { Yara }\end{array}$ & $\begin{array}{l}\text { Fertilización } \\
\text { ecologica }\end{array}$ & $\begin{array}{l}\text { Fertilización } \\
\text { orgánica }\end{array}$ & $\begin{array}{l}\text { Fertilización } \\
\text { química }\end{array}$ & $\begin{array}{c}\text { Sin } \\
\text { fertilización }\end{array}$ \\
\hline 0 días & $\begin{array}{c}\text { Yaravita Sinergise } \\
\mathrm{NP}+\mathrm{Zn} 100 \mathrm{cc} / 15 \mathrm{~kg} \\
\text { de semilla }\end{array}$ & $\begin{array}{l}\text { Yaravita Sinergise } \\
\mathrm{NP}+\mathrm{Zn} 50 \mathrm{cc} / 15 \mathrm{~kg} \\
\text { de semilla }\end{array}$ & Sin aplicación & Sin aplicación & $\begin{array}{l}\text { Sin } \\
\text { aplicación }\end{array}$ \\
\hline 8 días & $\begin{array}{c}\text { Yaravela Nitromag } \\
5 \mathrm{gr} / \text { planta }\end{array}$ & $\begin{array}{c}\text { Yaravela Nitromag } \\
\text { + Ful Humix 2,5gr/ } \\
\text { planta }\end{array}$ & Humus 5gr/planta & $\begin{array}{c}\text { Abono completo } \\
\text { 15-15-15 + Urea } \\
\text { 5gr/planta }\end{array}$ & $\begin{array}{l}\text { Sin } \\
\text { aplicación }\end{array}$ \\
\hline 15 días & $\begin{array}{c}\text { Yara Remital M. 17-6- } \\
\text { 18-2 5gr/planta }\end{array}$ & $\begin{array}{c}\text { Yara Remital 2,5gr/ } \\
\text { planta + Micor-9 } \\
100 \mathrm{cc} / 20 \text { lt de agua }\end{array}$ & $\begin{array}{l}\text { Fast2grown } 5 \mathrm{gr} / \\
\text { planta }\end{array}$ & Urea 5gr/planta & $\begin{array}{l}\text { Sin } \\
\text { aplicación }\end{array}$ \\
\hline 21 días & $\begin{array}{c}\text { Yara Zintrac } \\
100 \mathrm{cc} / 20 \text { lt de agua }\end{array}$ & $\begin{array}{c}\text { Yara Zintrac } \\
+ \text { Fast2grown } \\
50 \mathrm{cc} / 10 \text { lt de agua }\end{array}$ & $\begin{array}{c}\text { Humus + Grandsil } \\
100 \mathrm{cc} / 20 \text { lt de } \\
\text { agua }\end{array}$ & Urea 5gr/planta & $\begin{array}{l}\text { Sin } \\
\text { aplicación }\end{array}$ \\
\hline 40 días & $\begin{array}{l}\text { Yara Nitrodoble } \\
5 \mathrm{gr} / \text { planta }\end{array}$ & $\begin{array}{c}\text { Yara Nitrodoble + } \\
\text { Fossil Shell agro } \\
\text { 2,5gr/planta }\end{array}$ & $\begin{array}{c}\text { Micor-9 } 100 \mathrm{cc} / 2 \\
\text { lt de agua }\end{array}$ & Sin aplicación & $\begin{array}{l}\text { Sin } \\
\text { aplicación }\end{array}$ \\
\hline
\end{tabular}

Control de plagas: se realizó un control de RESULTADOS plagas haciendo una prevención de gusano de tierra (Phyllophaga) antes de la siembra con el uso de thiodicar y de gusano cogollero (Spodoptera frugiperda) a los 18 días.

Cosecha y recolección: se realizó la cosecha en estado seco a los 130 días.

En la Tabla 5, se exponen los resultados obtenidos de la experimentación 
Tabla 5. Resultados experimentación.

\begin{tabular}{ll}
\hline Mayor nivel de productividad & Peso de la parcela: $38,31 \mathrm{~kg}$ \\
\hline Mejor desarrollo morfológico & $\begin{array}{l}\text { Altura de planta: } 213,78 \mathrm{~cm} \\
\text { Diámetro: } 72 \mathrm{~mm} \\
\text { Longitud de hoja: } 101,58 \mathrm{~cm}\end{array}$ \\
\hline Mayor beneficio con un costo de producción & Por planta de $\$ 0,019$ y por ha de $\$ 1187,50$ \\
\hline Beneficio neto & Los tratamientos químicos con: $\$ 4423,30$ \\
\hline Altura de planta & El testigo con: $\$ 3769,08$ \\
\hline Menos beneficio bruto & Tratamiento ecológico: $209,2 \mathrm{~cm}$ \\
& Tratamiento orgánico: $206,2 \mathrm{~cm}$ \\
\hline
\end{tabular}

Costo económico estimado de producción y análisis de costo beneficio

La Tabla 6, presenta el costo de producción por planta según los tratamientos que se aplicaron, obteniendo un valor de 0,04 ctvs. por planta; en el tratamiento YARA y en el tratamiento sin aplicación siendo estos los rendimientos que marcaron la diferencia en cuanto al beneficio económico que se obtuvo entre los cinco tratamiento empleados durante la experimentación, alcanzando un porcentaje de retorno del $173 \%$ para el tratamiento YARA y $116 \%$ para el tratamiento sin aplicación, en tanto que los porcentajes de retorno de los tratamientos químico, ecológico y orgánico fueron del $140 \%, 56 \%$ y $49 \%$ respectivamente.

Los valores establecidos en la siguiente tabla representan el gasto obtenido por cada tratamiento aplicado en el proceso investigativo. 
Tabla 6. Costo de producción y relación costo beneficio.

\begin{tabular}{|c|c|c|c|c|c|}
\hline & $\begin{array}{l}\text { Fertilización } \\
\text { YARA }\end{array}$ & $\begin{array}{l}\text { Fertilización } \\
\text { Ecológica }\end{array}$ & $\begin{array}{l}\text { Fertilización } \\
\text { Orgánica }\end{array}$ & $\begin{array}{l}\text { Fertilización } \\
\text { Química }\end{array}$ & $\begin{array}{c}\text { Sin } \\
\text { aplicación }\end{array}$ \\
\hline Fertilizantes & $\$ 1,25$ & $\$ 13,25$ & $\$ 12,00$ & $\$ 0,95$ & $\$ 0,00$ \\
\hline Siembra & $\$ 2,00$ & $\$ 2,00$ & $\$ 2,00$ & $\$ 2,00$ & $\$ 2,00$ \\
\hline Riego & $\$ 4,00$ & $\$ 4,00$ & $\$ 4,00$ & $\$ 4,00$ & $\$ 4,00$ \\
\hline Mano de obra & $\$ 12,00$ & $\$ 12,00$ & $\$ 12,00$ & $\$ 12,00$ & $\$ 12,00$ \\
\hline Costo de semillas & $\$ 2,00$ & $\$ 2,00$ & $\$ 2,00$ & $\$ 2,00$ & $\$ 2,00$ \\
\hline Total, de costo por tratamiento & $\$ 21,25$ & $\$ 33,25$ & $\$ 32,00$ & $\$ 20,95$ & $\$ 20,00$ \\
\hline Costo de producción por planta (ctvs) & $\$ 0,04$ & $\$ 0,07$ & $\$ 0,06$ & $\$ 0,04$ & $\$ 0,04$ \\
\hline Costo de producción por ha & $\$ 2.656,25$ & $\$ 4.156,25$ & $\$ 4.000,00$ & $\$ 2.618,75$ & $\$ 2.500,00$ \\
\hline Peso promedio de granos por mazorca & 313,83 & 268,37 & 270,26 & 272,21 & 233,98 \\
\hline Peso de rendimiento por ha (gr) & 19614375 & 16773125 & 16891250 & 17013125 & 14623750 \\
\hline Peso de rendimiento por ha (kg) & 19614,38 & 16773,13 & 16891,25 & 17013,13 & 14623,75 \\
\hline Producción por ha (precio oficial) & $\$ 7.257,32$ & $\$ 6.206,06$ & $\$ 6.249,76$ & $\$ 6.294,86$ & $\$ 5.410,79$ \\
\hline $\begin{array}{l}\text { Ganancias (precio de producción- costo de } \\
\text { producción) }\end{array}$ & $\$ 4.601,07$ & $\$ 2.049,81$ & $\$ 2.249,76$ & $\$ 3.676,11$ & $\$ 2.910,79$ \\
\hline$\%$ de retorno & $173 \%$ & $49 \%$ & $56 \%$ & $140 \%$ & $116 \%$ \\
\hline
\end{tabular}

DISCUSIÓN

El tratamiento de YARA obtuvo una mayor productividad con relación al testigo sin aplicación ya que alcanzo un mayor porcentaje de retorno, tomando estos como rendimientos altos logrados en consideración a los demás tratamientos aplicados, en su blog "Una estrategia para aumentar hasta un 75\% el rendimiento del maíz" donde se menciona que a pesar de tener híbridos de maíz con alto potencial genético se debe enfocar en esquemas de fertilización que cumplan los objetivos nutricionales de la planta además se debe enfocar en aumentar la cantidad de los granos y su peso en cada mazorca, y donde al igual se establece que los cultivos de maíz sin fertilización son los más bajos en rendimientos representando de esta manera una baja retribución económica que no alcanza a solventar la inversión. (2)

En relación a los rendimientos de los tratamientos ecológicos y orgánicos para lograr la misma efectividad del tratamiento YARA se debería utilizar 13,5 veces más de fertilizante orgánico, (3), para lograr aportar la misma cantidad de nitrógeno a las plantas es necesario aplicar una tonelada de compost por hectárea la cual aportaría de 16 a $20 \mathrm{~kg}$ de nitrógeno representando una carga económica muy alta ya que una ha de maíz necesita de 200 a $250 \mathrm{~kg}$ solamente de nitrógeno (4). 
El uso de fertilizantes químicos a corto y mediano plazo dejan un efecto residual la cual limita que los futuros cultivos de maíz no alcancen su rendimiento óptimo, ya que estos bloquean la fácil absorción de los nutrientes contenidos en el suelo, viéndose esto reflejado en los resultados obtenidos durante esta investigación, donde se evidencia que el uso de fertilizantes químicos tienen un rendimiento bajo en relación al tratamiento YARA, (5), porque la disponibilidad de nutrientes de YARA es inmediata dando la cantidad necesaria de los mismos, permitiendo a su vez que las plantas puedan absorberlas de manera rápida y eficaz sin dejar un efecto residual en el suelo (3).

Los beneficios fundamentales de los productos YARA permiten favorecer la absorción de otros nutrientes como lo son Potasio, Magnesio y Calcio; y que a su vez ayudan a que la planta tenga una mayor tolerancia al estrés hídrico, ya que los Nitratos estimulan el crecimiento radicular, haciendo que la planta tenga mayor superficie de contacto, capacidad de búsqueda y exploración de agua en el suelo. (6).

\section{CONCLUSIONES}

Los resultados obtenidos permiten identificar que el fertilizante YARA, es el mejor tratamiento empleado en esta investigación en comparación con los demás tratamientos mostrando un mejor desarrollo morfológico del cultivo de maíz en cuanto a altura de planta, diámetro de tallo y longitud de hoja.

El tratamiento YARA, permitió incrementar el rendimiento del cultivo de maíz en un 57\% respecto al tratamiento sin fertilización (testigo), lo cual puede ser atribuible a su composición química a base de nitratos que permite una absorción directa por el cultivo.
La estimación económica efectuada permite indicar que el tratamiento YARA tiene un mayor porcentaje de retorno el cual es de $176 \%$, permitiendo indicar que este tratamiento tiene una mayor productividad con respecto a los otros tratamientos aplicados.

\section{REFERENCIAS BIBLIOGRÁFICAS}

1. ACCU WEATHER, 01 diciembre 2019. [En línea]. Available: https://www.accuweather. com/es/ec/cantagallo/1241952/weatherforecast/1241952.

2. Balaudo L. «Infocampo,» Agosto 2019. [En línea]. Available: https://www.infocampo.com. ar/una-estrategia-para-aumentar-hasta-un-75el-rendimiento-del-maiz/.

3. Pulido ID. «YARA,» 31 Octubre 2018. [En línea]. Available: https://www.yara.com.mx/noticiasy-eventos/noticias-mexico/mezclas-fisicas-vscomplejos-quimicos/.

4.BeistiguiAd. «Corfo»Diciembre2015. [En línea]. Available: https:/corfo.gob.ar/wp-content/ uploads/2015/12/Fertlizacionnitrogenadamaiz. pdf.

5. Alvarado A. Agronomía Costarricense 2009. [En línea]. Available: file://C:/Users/Fabian\%20 Merchan/Downloads/Dialnet-EfectoResidualD elFertilizanteFosfatadoAdicionadoAl-3022690. pdf.

6. YARA, «YARA,» 2021. [En línea]. Available: https://www.yara.com.mx/noticias-y-eventos/ noticias-mexico/ventajas-de-los-nitratos-en-laproductividad-de-tus-cultivos/. 